Casos Clínicos

Arch. Esp. Urol. 2010; 63 (5): 387-390

\section{NEFROSTOMÍA PERCUTÁNEA BILATERAL COMO TRATAMIENTO DE LA CISTITIS HEMORRÁGICA SEVERA}

Daniel Adolfo Pérez Fentes, Ana Puñal Pereira, Pablo Lorenzo González', Camilo García Freire, Serafín Novás Castro y Miguel Blanco Parra.

Servicio de Urología y Servicio de Anatomía Patológica?. Complejo Universitario de Santiago de Compostela.

Santiago de Compostela. La Coruña. España.

Resumen.- OBJETIVO: Presentar un caso de cistitis hemorrágica severa tratada exitosamente mediante nefrostomía percutánea bilateral.

MÉTODO: Presentamos el caso de una mujer de 67 años que presenta hematuria macroscópica monosintomática con coágulos.

RESULTADOS: Fracaso de los tratamientos conservadores habituales y desarrollo de vejiga coagulada. Evacuación endoscópica y electrocoagulación de las áreas sangrantes sin éxito. Dada la persistencia de hematuria y desarrollo de insuficiencia renal e inestabilidad hemodinámica se decide nefrostomía percutánea bilateral. A las 24 horas cesa la hematuria, la paciente recupera la estabilidad hemodinámica y no requiere nuevas transfusiones sanguíneas.

CONCLUSIONES: La realización de nefrostomía percutánea bilateral puede ser una opción útil en el tratamiento de la cistitis hemorrágica ante el fracaso de las medidas conservadoras habituales y como paso previo a la realización de otros tratamientos más invasivos.

Palabras clave: Cistitis hemorrágica. Nefrostomía percutánea

Summary.- OBJECTIVE: To report a case of severe hemorrhagic cystitis successfully treated by bilateral percutaneous nephrostomy.

METHODS: The case of a 67-year-old female patient who had monosymptomatic gross hematuria with clots is reported.

RESULTS: Standard conservative treatments failed and the patient developed a clot-retention plugged bladder. Endoscopic evacuation and electrocoagulation of bleeding areas was unsuccessful. Due to persistent hematuria and development of renal failure and hemodynamic instability, bilateral percutaneous nephrostomy was performed. At 24 hours, hematuria ceased, patient recovered hemodynamic stability, and no additional blood transfusions were required.

CONCLUSIONS: Bilateral percutaneous nephrostomy may be a valuable option for the treatment of hemorrhagic cystitis when standard conservative measures have failed and as a prior step to performance of other more invasive procedures.

Keywords: Hemorrhagic cystitis. Percutaneous nephrostomy.

CORRESPONDENCIA

Daniel Adolfo Pérez Fentes

Servicio de Urología

Complejo Universitario de Santiago de Compostela.

Travesía Choupana s/n.

15706. Santiago de Compostela.

La Coruña (España)

danielfentes@yahoo.es

Aceptado para publicar: 12 de marzo 2010

\section{INTRODUCCIÓN}

La cistitis hemorrágica $(\mathrm{CH})$ se define como un sangrado difuso, agudo o crónico, de la mucosa vesical, de etiología e intensidad variable. En función de la intensidad de su presentación clínica distinguiremos entre formas de $\mathrm{CH}$ leves, moderadas y severas (1). La mortalidad en la $\mathrm{CH}$ severa puede llegar hasta el $4 \%$, con una morbilidad asociada al cuadro mucho mayor.

Se han descrito numerosas opciones terapéuticas para la cistitis hemorrágica, con distinto grado de invasividad: hiperhidratación, irrigación vesical continua con 
suero, administración sistémica de agentes antifibrinolíticos, electrocoagulación endoscópica de los vasos sangrantes, instilaciones vesicales con distintos agentes (nitrato de plata, formol, aluminio o prostaglandinas), oxigenoterapia hiperbárica, tamponamiento vesical quirúrgico, embolización/ligadura de las arterias hipogástricas, derivación urinaria quirúrgica y cistectomía.

Aportamos un caso de cistitis hemorrágica severa, resuelta exitosamente mediante realización de nefrostomía percutánea bilateral, tras el fracaso de los tratamientos conservadores habituales, sin requerir la aplicación de medidas quirúrgicas de mayor invasividad.

\section{CASO CLÍNICO}

Describimos el caso de una mujer de 67 años, sin antecedentes urológicos de interés, que presenta un cuadro de hematuria macroscópica monosintomática con coágulos de 2 días de evolución. Inicialmente se decide tratamiento conservador con sondaje vesical y circuito de lavado con suero fisiológico. A las 24 horas la paciente está anémica e hipotensa, requiriendo aporte de aminas vasoactivas y politransfusión de concentrados hemáticos. Se realiza TC abdómino-pélvico que informa de la existencia de una vejiga completamente ocupada por un gran coágulo vesical y ureterohidronefrosis bilateral moderada secundaria (Figura 1).

Se interviene a la paciente endoscópicamente evacuando aproximadamente 1 litro de coágulos. La mucosa vesical está hiperémica, con sangrado difuso, sin evidencia de lesiones endovesicales. Se procede a la electrocoagulación de varias áreas con sangrado activo. La paciente ingresa en la Unidad de Cuidados Intesivos con intubación orotraqueal y tratamiento de soporte con aminas vasoactivas. Se añade al tratamiento ácido ami-

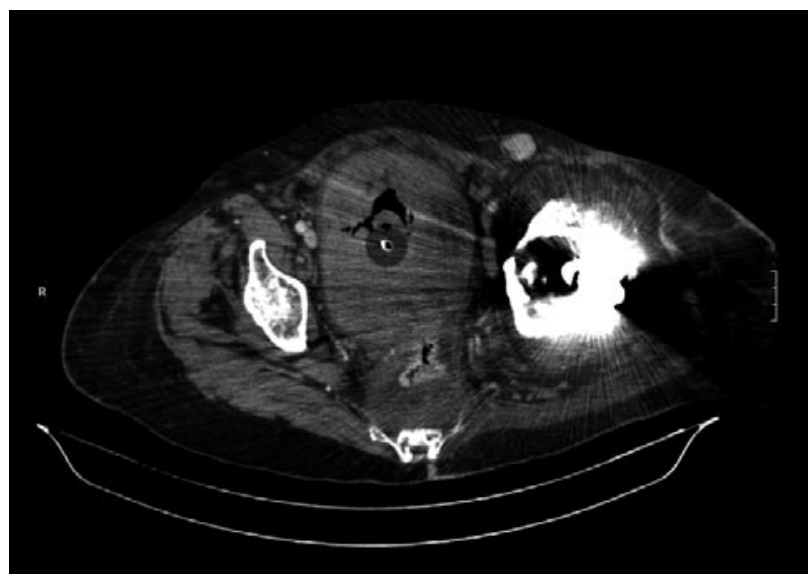

FIGURA 1. TC abdómino-pélvico. Vejiga coagulada. nocaproico intravenoso. A las 24 horas la vejiga está nuevamente coagulada y la paciente continúa anémica e inestable hemodinámicamente, pese a haber recibido transfusión de 10 concentrados hemáticos.

Ante esta situación decidimos realizar nefrostomía percutánea bilateral (NPC) con la doble intención de solventar el problema obstructivo producido por la ocupación vesical e intentar reducir el sangrado a ese nivel.

Se realiza nefrostomía percutánea bilateral eco y radiodirigida, bajo anestesia general, en posición de Valdivia, colocando sendos catéteres $8 \mathrm{Ch}$ a través de cáliz inferior, sin incidencias (Figura 2).

A las 24 horas de la realización de la NPC bilateral la paciente mejora significativamente, cesando la hematuria y no requiriendo nuevas transfusiones sanguíneas. Dos días después se retiran las aminas vasoactivas y la intubación orotraqueal, siendo dada de alta de la Unidad de Cuidados Intensivos al cuarto día.

Una vez superado el cuadro agudo, se programa nueva revisión endoscópica vesical con toma de biopsias. El estudio anátomo-patológico evidenció como única alteración reseñable la presencia de un material eosinófilo dispuesto alrededor de los vasos sanguíneos de la submucosa. Esta sustancia se teñía con la tinción de Rojo Congo adquiriendo color verde manzana bajo la exposición de luz polarizada con birrefringencia, lo que confirmó que se trataba de amiloide (Figura 3). El estudio inmunohistoquímico de la lesión con anticuerpos monoclonales, (clon $\mathrm{mcl}$ ), específicos frente a la

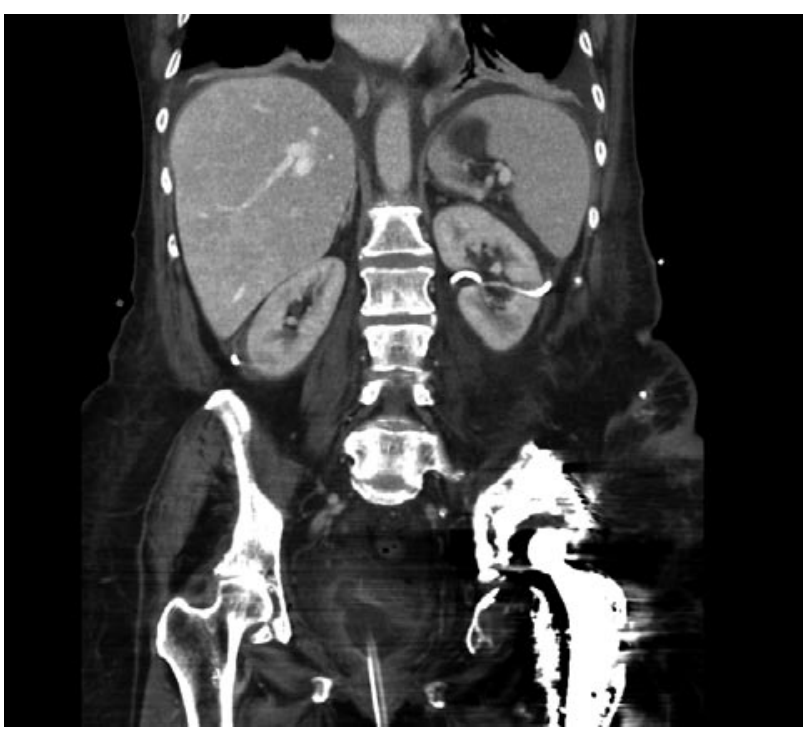

FIGURA 2. TC abdómino-pélvico. NPC a través de cáliz inferior. 
proteína AA del amiloide resultó positiva permitiendo el diagnóstico de Amiloidosis vesical secundaria (Tipo AA). El postoperatorio transcurre sin incidencias, retirándose las nefrostomías a los 20 días y siendo dada de alta al mes.

Tras 6 meses de seguimiento, la paciente no ha vuelto a presentar hematuria y está pendiente de estudios para descartar afectación sistémica por la amiloidosis.

\section{DISCUSIÓN}

La cistitis hemorrágica se define como un sangrado difuso, agudo o crónico de la mucosa vesical, de etiología e intensidad variable. Entre las causas más frecuentes de $\mathrm{CH}$ destacan el tratamiento quimioterápico con ciclofosfamida y agentes derivados y la radioterapia pélvica. Otras causas posibles de $\mathrm{CH}$ incluyen antibióticos (penicilinas), antiinflamatorios no esteroideos, toxinas ambientales (anilinas), enfermedades infecciosas (virales, bacterianas, fúngicas y parasitosis) y enfermedades sistémicas (enfermedades del colágeno, amiloidosis) (2).

En función de la severidad de su presentación clínica, DeVries (1) propone una clasificación de la $\mathrm{CH}$ en formas leves, moderadas y severas. Así, la $\mathrm{CH}$ severa se define como una hemorragia que requiere transfusión de 6 o más concentrados hemáticos para mantener la estabilidad hemodinámica del paciente, debido a su duración y a la refractariedad a la aplicación de medidas terapéuticas conservadoras habituales, como la irrigación vesical continua o la administración de agentes antifibrinolíticos. La cistitis hemorrágica severa tiene una mortalidad del $4 \%$, con una morbilidad asociada mucho mayor.

En el tratamiento de la cistitis hemorrágica se han propuesto numerosas opciones terapéuticas. Algunos autores han propuesto algoritmos de tratamiento, de menor a mayor invasividad, en función de la severidad de los síntomas y de la refractariedad del cuadro (2). Entre las opciones terapéuticas conservadoras destacan la hiperhidratación, la irrigación vesical continua con evacuación manual de coágulos endovesicales y la administración de agentes antifibrinolíticos. Ante el fracaso de las medidas conservadoras habituales, puede recurrirse a la evacuación endoscópica de coágulos seguida de electrocoagulación de los vasos sangrantes. Otras medidas terapéuticas son la oxigenoterapia hiperbárica, bien tolerada por el paciente, y la instilación endovesical de distintos agentes (nitrato de plata, aluminio, prostaglandinas o formol), con un perfil de efectos secundarios considerable $(3,4)$. Como escalón final, ante la refractariedad del cuadro, se describen como opciones válidas: la embolización/ligadura de ambas arterias hipogástricas (5), la realización de cistotomía y tamponamiento vesical (6), la realización de una derivación urinaria quirúrgica (7) y, en último caso, la cistectomía (8).
En nuestro caso, dada la persistencia del sangrado pese a la aplicación de las medidas conservadoras habituales y la evacuación endoscópica de coágulos con electrocoagulación de los vasos sangrantes, optamos por una alternativa de invasividad intermedia: la realización de una derivación urinaria mediante nefrostomía percutánea bilateral.

La nefrostomía percutánea es una técnica endourológica habitual, de fácil realización y que presenta una baja mortalidad $(<1 \%)$ y morbilidad $(<10 \%)$. Permite una derivación urinaria temporal y reversible, con menor invasividad que las derivaciones urinarias quirúrgicas (ureterostomía cutánea, ureterosigmoidostomía, ureteroileostomía cutánea). Nosotros optamos por realizar ambas nefrostomías percutáneas en posición supina, siguiendo la descrita por Gabriel Valdivia en 1987 (9), al igual que lo hacemos en nuestro Centro para la nefrolitotomía percutánea. La posición supina ofrece frente al prono diversas ventajas: técnicas, para el paciente, y anestesiológicas; sobre todo en pacientes como el tratado, con inestabilidad hemodinámica y ventilación mecánica.

Hasta nuestro conocimiento sólo existen 2 artículos en la literatura médica internacional que refieran su empleo como tratamiento de la cistitis hemorrágica severa $(10,11)$.

La utilidad de la realización de una derivación urinaria mediante nefrostomía percutánea bilateral en el tratamiento de la $\mathrm{CH}$ severa es doble. Por un lado, al disminuir el flujo urinario hacia la vejiga se evita la ruptura de vasos sanguíneos mucosos friables por sobredistensión vesical. Por otro lado, disminuye los niveles locales de urokinasa, enzima fibrinolítica sintetizada por el riñón y liberada por la orina, favoreciendo la hemostasia. Esta

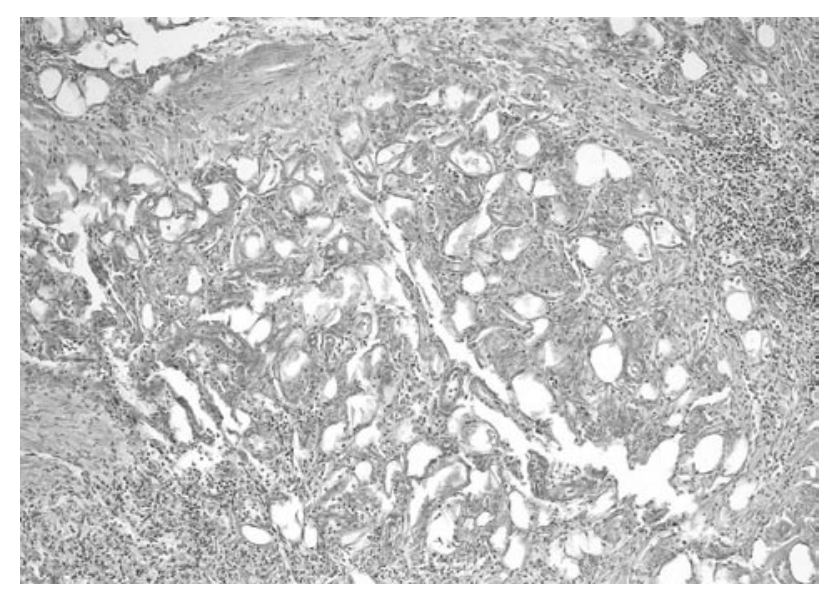

FIGURA 3. Tinción Rojo Congo demostrando depósitos de amiloide alrededor de los vasos sanguíneos. 
teoría se ve avalada por la efectividad de la administración local o sistémica de fármacos antifibrinolíticos lácido épsilon-aminocaproico, ácido tranexámico y aprotinina). Sin embargo, para confirmar esta hipótesis se necesitan estudios en los que se cuantifique la excreción urinaria vesical de urokinasa en pacientes antes y después de realizar la derivación urinaria.

Algunos autores proponen asociar al procedimiento percutáneo la oclusión ureteral con cáteter balón. En nuestra opinión, este procedimiento no es necesario, pues el flujo urinario se establece de modo casi total a través del catéter de nefrostomía, al presentar una menor resistencia al mismo que el uréter (12).

En nuestro caso, dada la persistencia de la cistitis hemorrágica y estando la paciente hemodinámicamente inestable, decidimos realizar una medida de menor invasividad que las quirúrgicas descritas previamente. Con la realización de nefrostomía percutánea bilateral conseguimos evitar la sobredistensión vesical, mejorar la función renal de la paciente y el cese del sangrado a las 24 horas. Las nefrostomías percutáneas fueron retiradas posteriormente, sin secuelas para la paciente. Esto añade, además de la baja morbi-mortalidad asociada a la técnica, la ventaja de su reversibilidad, frente a las técnicas quirúrgicas abiertas, que quedarían reservadas para el escalón final dentro del algoritmo terapéutico de la cistitis hemorrágica.

\section{CONCLUSIONES}

La realización de nefrostomía percutánea bilateral es una técnica mínimamente invasiva y reversible, que puede ser una opción útil para el tratamiento de la cistitis hemorrágica severa. Proponemos su inclusión dentro del algoritmo terapéutico de la cistitis hemorrágica ante el fracaso de medidas más conservadoras y como paso previo a la realización de procedimientos quirúrgicos más invasivos.

\section{BIBLIOGRAFÍA y LECTURAS RECOMENDADAS (*lectura de interés $y$ ** lectura fundamental)}

*1. Devries CR, Freiha FS. Hemorrhagic cystitis: a review. J Urol, 1990; 143(1): 1-9.

**2. Traxer O, Desgrandchamps F, Sebe P, Haab F, Le duc A, Gattegno B, Thibault P. Cystite hémorragique: étiologie et traitement. Prog Urol, 2001; 11(4): 591-601.

3. Perazella M, Brown E. Acute aluminum toxicity and alum bladder irrigation in patients with renal failure. Am J Kidney Dis, 1993; 21(1): 44-46.

4. Donahue LA, Frank IN. Intravesical formalin for hemorrhagic cystitis: analysis of therapy. J Urol, 1989; 141(4): 809-812.
5. Mackie S, Lam T, Rai B, Nabi G, Mcclinton S. "Management of urological hemorrhage and the role of transarterial angioembolization". Minerva Med, 2007; 98(5): 511-524.

6. Andriole GL, Yuan JJ, Catalona WJ. Cystotomy, temporary urinary diversión and bladder packing in the management of severe cyclophosphamideinduced hemorrhagic cystitis. J Urol, 1990; 143(5): 1006-7.

7. Pomer S, Karcher G, Simon W. Cutaneous ureterostomy as last resort treatment of intractable haemorrhagic cystitis following radiation. Br J Urol, 1983; 55(4): 392-4.

8. Hassan Z, Remberger M, Elbander M et al. Hemorrhagic cystitis: a retrospective single-center survey. Clin Transplant, 2007; 21 (5): 659-667.

*9. Valdivia-Uría JG, Lanchares E, Villaroya S, Taberner J, Abril G, Aranda JM. Nefrolitotomía percutánea: Técnica simplificada (nota previa). Arch Esp Urol, 1987; 40(3): 177-180.

**10. Zagoria RJ, Hodge RG, Dyer RB, Routh WD. Percutaneous nephrostomy for treatment of intractable hemorrhagic cystitis. J Urol, 1993; 149(6): 1449-51.

**11. Sneiders A, Pryor JL. Percutaneous nephrostomy drainage in the treatment of severe hemorrhagic cystitis. J Urol, 1993; 150(3): 966-7.

12. Saltzstein DR, Orihuela E, Reed J. Ballon ureteral occlusion: a new reversible technique in the management of ureteral fistulas. J Urol, 1992; 147(2): 407-8.

13. Nurmi MJ, Walls J. Secondary amyloidosis of the bladder: a cause of massive hematuria. J Urol, 1987; 138:44-5.

14. Begara Morillas F, Silmi Moyano A, Espinosa Fernández $\mathrm{B}$, et al. Amiloidosis vesical en un paciente con sospecha de tumor vesical. Arch Esp Urol, 1994; 47(2):176-9. 